Invited Talk I

\title{
Microwave Characterization of Materials for Telecommunication Systems
}

\author{
Janina Mazierska
}

\begin{abstract}
:
Novel low loss materials are needed to achieve miniaturization and further advances in wireless communications with precisely known microwave properties. Measurements of complex permittivity of low loss dielectric materials (especially anisotropic) in a wide range of temperatures still represent a challenging issue. Accuracy of surface resistance tests of superconducting materials can be low in some cases, and characterization of metamaterials is done only for one polarization and one incident angle, what is rather unsatisfactory. Accuracy of measurements is also determined by accuracy in determination of unloaded Qo-factor of test fixtures. Hence progress in microwave characterization of novel materials is also necessary. In this presentation a review of test resonators and a precise technique to measure the Qo-factor will be given. Also measurement results of complex permittivity of several low loss dielectrics and High Temperature superconducting materials will be shown.
\end{abstract}

978-1-4244-5076-3/09/\$26.00 C2009 IEEE 